\title{
Modelo de identificación y caracterización de tramos de carreteras sin accidentes (tramos blancos). Aplicación a la red de autopistas de peaje españolas
}

\author{
Model for the identification and characterization of road sections with- \\ out accidents (white sections). Application in the Spanish toll motorways \\ network
}

E. de la Peña-González ${ }^{(*)}$, A. Zaragoza-Ramírez ${ }^{(*)}$

\section{RESUMEN}

El planteamiento tradicional de análisis de la accidentalidad en carretera pasa por la consideración de herramientas paliativas, como son la identificación y gestión de los puntos negros o tramos de concentración de accidentes, o preventivas, como las auditorías o inspecciones de seguridad vial. En este artículo se presenta un complemento a estas herramientas, desde una perspectiva novedosa: la consideración de los tramos donde, bajo unas condiciones similares a las de otros tramos y una similar exposición al riesgo, no se producen accidentes (tramos blancos). Se resume el modelo que se ha calibrado para las autopistas de peaje españolas, identificando los parámetros específicos de la geometría de la vía y del tráfico que más influencia tienen en el hecho de que en algunos tramos de suficiente longitud, no se produzcan accidentes durante un periodo significativo de tiempo: el tráfico de pesados, al ancho de calzada y la velocidad de circulación.

Palabras clave: Accidente; seguridad vial; modelo; carretera; autopista.

\section{ABSTRACT}

The traditional approach to infrastructural safety follows the consideration of mitigation tools, such as the identification and management of black spots or road sections with high accident concentration, or preventing tools, as road safety audits and inspections. A complement to these tools is presented in this paper, from a new perspective: the consideration of road sections where, under similar conditions and similar risk exposure, there are no accidents (white road sections). A model has been calibrated to the Spanish toll motorways network, identifying the specific geometric parameters and traffic characteristics which have the highest influence in the occurrence of white sections with sufficient length, without accidents during a significant period of time: heavy vehicles traffic volume, with of the road section and speed.

Keywords: Accident; road safety; model; highway; motorway.

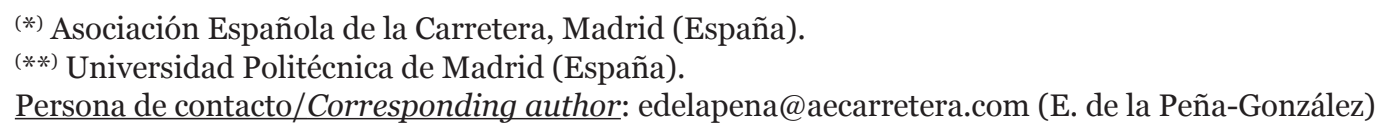

Cómo citar este artículo/Citation: de la Peña-González, E., Zaragoza-Ramírez, A. (2015). Modelo de identificación y caracterización de tramos de carreteras sin accidentes (tramos blancos). Aplicación a la red de autopistas de peaje españolas. Informes de la Construcción, 67(540): e117, doi: http://dx.doi.org/10.3989/ic.14.135.

Licencia / License: Salvo indicación contraria, todos los contenidos de la edición electrónica de Informes de la Construcción se distribuyen bajo una licencia de uso y distribución Creative Commons Reconocimiento no Comercial 3.o. España (cc-by-nc). 


\section{LA SEGURIDAD EN LAS INFRAESTRUCTURAS VIARIAS}

Se estima que cada año mueren en el mundo 1,24 millones de personas en accidentes de tráfico, y al menos otros 50 millones resultan heridos, según la Organización Mundial de la Salud (OMS) (1).

En el caso de la Unión Europea, si bien los Estados Miembros son autónomos a la hora de aplicar sus políticas de seguridad vial, deben poner en práctica las Directivas y recomendaciones europeas, para homogeneizar las actuaciones y alcanzar objetivos comunes, como el recogido en el Libro Blanco del Transporte de la Unión Europea (2001), en el que se instaba a los Estados Miembros a reducir el número de víctimas mortales un 50 \% en el periodo 2001-2010 (2). La nueva estrategia europea de seguridad vial para la década 2011-2020 ha vuelto a establecer como objetivo común la reducción de otro $50 \%$ el número de fallecidos en este periodo (3).

En el contexto español, durante los últimos años se ha experimentado una notable reducción en las cifras de accidentalidad, que ha permitido alcanzar e incluso superar el objetivo europeo de disminuir las cifras de fallecidos en un $50 \%$ en la década 2000-2010.

\section{EVOLUCIÓN DEL TRATAMIENTO DE LA ACCIDENTALIDAD EN CARRETERA}

La preocupación por los accidentes vinculados al transporte, no ya solo desde el punto de vista humano y social, sino también desde la perspectiva del legislador, se activa a partir del siglo dieciocho. Desde entonces, no ha habido un único esquema conceptual para abordar los accidentes, sino que han convivido diferentes teorías que han convivido en el tiempo y en el espacio (4). Desde el concepto de la irresponsabilidad o privacidad de los accidentes de tráfico, al paso posterior de la teoría normativa - donde las normas salvaguardan la seguridad de los implicados- y a su evolución hacia la teoría de la dispersión -que se centra en actuaciones paliativas-, el ritmo de cambio es muy lento. Las últimas décadas han significado una auténtica revolución, al incluir las teorías de la planificación y de la satisfacción y plantear así, las posibilidades de control del problema y de gestión del mismo.

Los primeros pasos en el tratamiento de la accidentalidad en la carretera basada en recogida de datos reales de accidentes para su análisis se remontan al siglo XIX, aunque evolucionaron significativamente en el siglo XX. No ha sido hasta hace relativamente poco tiempo cuando se ha generalizado el análisis detallado y completo de accidentes, implantando las estrategias de identificación y gestión de puntos negros.

El concepto de punto negro ha sido superado por el de Tramo de Concentración de Accidentes (TCA), que se utiliza, con algunos matices diferenciadores, en las Administraciones de Carreteras; considera como emplazamientos de riesgo aquéllos que presentan una accidentalidad significativamente superior a la media para un determinado tipo de vías.

Es preciso destacar que el número de accidentes que se producen en un tramo de carretera varía de manera aleatoria cada año; efectos como la regresión a la media, por el que los niveles de accidentalidad en un tramo de carretera considerado como de mayor riesgo que la media tienden a descender en los años siguientes, son consecuencia de esa aleatoriedad. Asimismo, se producen circunstancias como la migración de accidentes, concepto que hace referencia al frecuente aumento en el número de accidentes en los tramos cercanos al punto negro tratado. La consideración de periodos amplios de estudio, como el utilizado en este análisis, amortigua, en buena medida, estos efectos, que perturban el análisis estadístico.

Con el fin de establecer procedimientos que permitan asegurar un nivel de seguridad elevado y homogéneo en toda la Red Transeuropea de Carreteras, la Directiva 2008/96/CE (5) exige la aplicación sistemática de evaluaciones de impacto de seguridad vial, procedimientos de gestión de la seguridad de la red de carreteras, auditorías e inspecciones de seguridad vial. La mencionada Directiva fue incorporada al ordenamiento jurídico español por medio del Real Decreto 345/2011 (6).

\section{PARÁMETROS DE LA VÍA, EL TRÁFICO Y SU ENTORNO QUE INFLUYEN EN LA SEGURIDAD VIAL}

La investigación en los parámetros de la vía que más influyen en la accidentalidad ha sido muy amplia. Mohamedshah y otros (7) formularon un modelo para la determinación de la tasa de implicación de camiones en accidentes por kilómetro y año, en función de la intensidad media diaria de camiones y otros vehículos. Garber y Ehrhart (8), determinaron la influencia de la velocidad media, desviación estándar de la velocidad, flujo por carril, ancho de carril y arcén con la tasa de accidentes, mediante análisis multivariante, regresión robusta y regresión lineal. Buaer y Hardwood (9) evaluaron la influencia del tráfico en las intersecciones y en carriles de aceleración, concluyendo que un bajo porcentaje de accidentes está relacionado con la geometría, siendo el tráfico el factor más influyente. Karlaftis y Golías (10) identificaron la Intensidad Media Diaria como la variable que mejor explica los accidentes en carreteras de varios carriles, así como la mejora de la seguridad ante la existencia de medianas y control de accesos. Hiselius (11) valoró la elevada influencia del tráfico de vehículos pesados en las tasas de accidentalidad, en comparación con la influencia del tráfico total. Arenas (12) concluyó que la composición del tráfico y su heterogeneidad determina diferencias en los niveles de accidentalidad. Deubleien (13) realizó un análisis bayesiano para determinar la influencia de numerosos parámetros en el riesgo de sufrir un accidente, concluyendo que el tráfico y la velocidad presentaban una fuerte influencia en numerosas hipótesis.

Autores como Hadi (14), Karlafatis y Tarko (15), Abdel-Aty y Radwan (16) o Xie (17), analizaron la influencia de la sección transversal en el número de accidentes que se producen en las vías.

La influencia de elementos del trazado horizontal y vertical como el radio de las curvas o las pendientes, han sido analizadas por autores como Vogt y Bared (18), Persaud (19), Chang y Chen (20), Berhanu (21), con resultados variados.

\section{UNA NUEVA APROXIMACIÓN AL ESTUDIO DE LA ACCIDENTALIDAD: EL ANÁLISIS DE LOS TRAMOS BLANCOS}

\subsection{Antecedentes}

El concepto de «tramo blanco» fue desarrollado por primera vez en el año 2001 por iniciativa de la Asociación Española 
de la Carretera y el Instituto de Seguridad Vial de la Fundación MAPFRE (22), en un trabajo en el que participaron los autores. Se pretendía identificar los tramos de la red convencional de carreteras del Estado en España, de más de 25 kilómetros, donde no se hubieran producido accidentes con víctimas durante un periodo relevante de tiempo, que se fijó en cinco años; se identificaron más de 3.000 kilómetros de tramos blancos (23).

Asimismo, en abril de 2010, la Comisión Europea, a través de la Dirección General de Movilidad y Transporte, aprobó una subvención para la realización del proyecto WHITEROADS (White Spots in the Trans-European Road Network - A positive approach to road safety), que se desarrolló hasta 2013 (24). El proyecto permitió definir e identificar 982 Tramos Blancos en la Red Transeuropea de Carreteras en los 27 Estados Miembros de la Unión Europea, en su mayor parte vías de gran capacidad.

La metodología de tramos blancos pretende identificar los tramos de vía que están experimentando un mejor comportamiento desde la perspectiva de la accidentalidad, y analizar sus características de diseño, equipamiento, tráfico, etcétera, con el objetivo de buscar pautas comunes para el diseño, construcción y explotación de carreteras con numerosos tramos blancos (carreteras blancas).

El interés del desarrollo de una nueva metodología para investigar las características de los tramos de carreteras en los que no se producen accidentes durante un tiempo suficientemente amplio, si se comparan con tramos con características generales similares, radica en la suposición de que existen parámetros específicos, no sólo de diseño viario, sino también de tráfico, que tienen influencia en el hecho de que se produzcan o no accidentes.

En el caso de las autopistas de peaje, donde se ha realizado una primera aproximación para la descripción del modelo, es preciso señalar que se trata de una red viaria con mayor homogeneidad que en el caso de las vías de un solo carril por sentido, y en principio, con una mayor coherencia en el diseño. La identificación de tramos blancos, de suficiente longitud (parámetro que debe definirse), pone de manifiesto que, bajo una situación de coherencia en el diseño en las vías de más alta calidad de una red, en algunos casos se generan situaciones de mayor seguridad que en otras.

\subsection{Definición genérica de Tramo Blanco}

De acuerdo con los trabajos anteriormente realizados en la identificación de tramos sin accidentes, puede plantearse la siguiente definición genérica del tramo blanco: «tramo de carretera de una determinada longitud donde no se han producido accidentes durante un periodo determinado de tiempo».

La definición genérica plantea varias incógnitas que es preciso resolver para continuar con la investigación: la clasificación de las vías a considerar en el análisis, de manera que se analicen conjuntamente tramos con características homogéneas; la definición de la longitud de referencia de carretera sin accidentes para ser considerado tramo blanco, de manera que sea estadísticamente representativo; la determinación del periodo de tiempo para el análisis; y la decisión acerca del tipo de accidente que se va a considerar.
En relación a la primera de las cuestiones, parece oportuno realizar una clasificación de las vías en función de su geometría (separando vías de gran capacidad de vías convencionales), su funcionalidad y sus niveles de tráfico total y de vehículos pesados. Para esta análisis se considerará una categorización de autopistas de peaje establecida por la Dirección General de Tráfico en el marco del proyecto europeo de investigación EASYWAY (25), que clasifica las vías de los países de la Unión Europea según sus problemas de congestión y seguridad; asimismo, se ha realizado una clasificación por niveles de tráfico valorando la Intensidad Media Diaria en los tramos analizados, de manera que las vías analizadas se caractericen por niveles similares de exposición al riesgo.

Acerca de la definición de la longitud de referencia, debe realizarse a partir de la muestra estadística de tramos sin accidentes para una determinada categoría de vía, garantizando una buena representatividad estadística. Siguiendo los criterios habituales de análisis de tráfico y accidentalidad, se considera que una longitud de referencia igual al percentil 95 de las longitudes de los tramos sin accidentes es suficientemente representativa.

El marco temporal se establece de acuerdo a los análisis de accidentalidad que se realizan habitualmente en carreteras, donde se recomienda utilizar al menos datos de tres años, e idealmente, de un periodo de cinco años. En el análisis que se presenta en este artículo, el periodo de estudio ha sido 20062010, ambos incluidos.

En relación al tipo de accidente a valorar, es preciso decidir entre la consideración de accidentes con víctimas mortales, heridos graves y heridos leves. Se decide considerar únicamente los accidentes con víctimas mortales y heridos graves, para no aumentar excesivamente el tamaño de la muestra con accidentes de impacto reducido.

Por último, es importante añadir un requisito adicional a los tramos blancos, relacionado con la peligrosidad total de las vías en las que se encuentran; de esta manera, no parece apropiado considerar un tramo blanco que se localiza en una carretera con una peligrosidad muy superior a la media para la categoría de vías a la que pertenece; adicionalmente, no se consideran tramos blancos los tramos sin accidentes que se localizan muy próximos a tramos de elevada accidentalidad. La presencia de tramos blancos debe estar asociada a una reducción en la peligrosidad media de la vía respecto a los valores medios de cada categoría.

Así, la definición genérica de tramo blanco, para el estudio que se presenta en este estudio, figura a continuación:

«Tramo de vía, de longitud igual al percentil 95 de las longitudes de tramos sin accidentes con heridos graves o víctimas mortales durante el periodo 2006-2010 (para una misma categoría de vía, definida según la tipología de vía y un nivel similar de exposición al riesgo), en el que no se han producido accidentes con heridos graves o víctimas mortales en ese periodo, que cumplan, además, con el criterio de no localizarse entre tramos de alto riesgo y no situarse en una vía cuya peligrosidad (definida como la relación entre los accidentes con víctimas y el tráfico en millones de vehículos-kilómetro) sea superior a la media de esa categoría». 


\section{DEFINICIÓN DEL MODELO DE IDENTIFICACIÓN DE TRAMOS BLANCOS EN AUTOPISTAS DE PEAJE}

La definición del modelo, en el caso de las autopistas de peaje, pasa por las fases que se detallan a continuación.

\subsection{Categorización de vías}

La categorización de las autopistas de peaje se ha realizado de acuerdo a los datos facilitados por la Dirección General de Tráfico en el contexto del proyecto Easyway, mencionado anteriormente. Los trabajos desarrollados a lo largo de este estudio se han centrado en una parte significativa de la red de autopistas de peaje (1542 kilómetros en cada sentido de circulación), han permitido identificar un conjunto de tramos blancos, en diferentes categorías de vías. Se han considerado las autopistas que se incluyen en la Tabla 1.

Se han considerado todas las autopistas de peaje de España a excepción de la AP-7, debido a una kilometración duplicada de algunos tramos.

En el caso de las autopistas de peaje españolas, no se apreció distinción entre el tráfico de vehículos pesados en las categorías $\mathrm{B}$ y $\mathrm{C}$.

\subsection{Identificación de tramos sin accidentes y definición de longitud de referencia de tramo blanco}

La investigación continúa realizando un análisis de las longitudes de tramos sin accidentes en las autopistas mencionadas anteriormente, a partir de los datos de accidentalidad para el periodo 2006-2010, facilitados por la Dirección General de Tráfico. Los sentidos de las vías se analizan de manera separada en vías de dos o más carriles por calzada, como es el caso de las autopistas de peaje.
Así, se consideran las siguientes longitudes de referencia (percentil 95 de las longitudes de tramos sin accidentes), dentro de cada categoría de vía, para que un tramo sin accidentes pueda ser considerado como un tramo blanco, si se igual o supera esta longitud:

- Categoría A: 42,4 kilómetros

- Categoría B: 14,9 kilómetros

- Categoría C: 14,2 kilómetros

- Categoría D: 5,4 kilómetros

El hecho de que la longitud de referencia del tramo blanco prácticamente coincida para las categorías de vías $\mathrm{B}$ y $\mathrm{C}$ apunta nuevamente a la unión de ambas categorías.

La consideración de tramos blancos con longitud igual o superior a la longitud de tramo blanco de referencia incluida anteriormente da lugar a la identificación de 35 potenciales tramos blancos en la red de autopistas de peaje. Como se ha indicado anteriormente, es preciso descartar tramos blancos en vías cuya peligrosidad sea significativamente superior a la de la media de su categoría (se asume que este valor sea el doble del valor medio de peligrosidad de las vías de su categoría; por este motivo se descartan 6 potenciales tramos blancos); asimismo, no se admite la presencia de un verdadero tramo blanco si éste se encuentra entre dos tramos de alta peligrosidad (por este motivo se descarta 1 potencial tramo blanco).

De esta manera, se asume que un verdadero tramo blanco hace que el tramo contiguo de la vía siga teniendo una peligrosidad inferior a la media y la vía de la que forma parte sea, en términos globales, más segura que las carreteras de su misma categoría. Los 28 tramos blancos identificados se distribuyen en las diferentes categorías de vías según se muestra a continuación:

- Categoría A: 1 tramo blanco (en la R4)

- Categoría B: 23 tramos blancos (en la AP2, AP36, AP51, AP53, AP61, AP68 y AP71)

Tabla 1. Autopistas consideradas en el análisis por categorías (Fuente: Easyway).

\begin{tabular}{|c|c|c|}
\hline AUTOPISTA & LONGITUD & CATEGORÍA \\
\hline $\mathrm{AP}_{41}$ & 65 & \multirow{5}{*}{$\begin{array}{l}\text { A: Autopista periurbana, sin/con tráfico de pesados } \\
\text { relevante }\end{array}$} \\
\hline $\mathrm{R} 3$ & 27 & \\
\hline R4 & 51 & \\
\hline $\mathrm{R} 5$ & 30 & \\
\hline $\mathrm{R} 2$ & 59 & \\
\hline $\mathrm{AP} 2$ & 227 & \multirow{8}{*}{$\begin{array}{l}\text { B: Autopista con problemas de tráfico estacionales sin tráfico } \\
\text { de pesados relevante (sin/con problemas climatológicos) }\end{array}$} \\
\hline $\mathrm{AP}_{36}$ & 143 & \\
\hline AP68 (PK 0-77) & 77 & \\
\hline $\mathrm{AP}_{71}$ & 37 & \\
\hline $\mathrm{AP}_{4}$ & 90 & \\
\hline $\mathrm{AP}_{51}$ & 21 & \\
\hline AP61 & 25 & \\
\hline $\mathrm{AP}_{53}$ & 55 & \\
\hline AP66 & 73 & \multirow{5}{*}{$\begin{array}{l}\text { C: Autopista con problemas de tráfico estacionales } \\
\text { con tráfico de pesados relevante (sin/con problemas } \\
\text { climatológicos) }\end{array}$} \\
\hline $\mathrm{AP9}$ & 159 & \\
\hline $\mathrm{AP} 9 \mathrm{~F}$ & 34 & \\
\hline AP6 (PK 53-109) & 56 & \\
\hline AP68 (PK 77-291) & 214 & \\
\hline AP6 (PK 39-53) & 14 & \multirow{2}{*}{$\begin{array}{l}\text { D: Autopista con problemas de tráfico diarios con tráfico de } \\
\text { pesados relevante (sin/con problemas climatológicos) }\end{array}$} \\
\hline $\mathrm{AP} 1$ & 75 & \\
\hline
\end{tabular}


- Categoría C: 4 tramos blancos (en la AP9 y AP68)

- Categoría D: o tramos blancos

Dada la escasa representatividad de tramos blancos en la categoría $\mathrm{A}$, se decide continuar el análisis en las vías de categorías $\mathrm{B}$ y C; en el análisis se incorporan tanto las vías que presentan tramos blancos, citadas anteriormente, como aquéllas en las que no se han localizado tramos blancos, según el criterio definido de manera provisional (es el caso de la AP66, $\mathrm{AP}_{4}, \mathrm{AP} 9 \mathrm{~F}$ y AP6, entre los puntos kilométricos 53 y 109).

\subsection{Caracterización de variables y datos para el modelo}

Para cada una de las autopistas consideradas en el análisis, se ha realizado una caracterización de las variables del estudio, que se enumeran posteriormente, considerando una tramificación delas vías de acuerdo a los siguientes criterios:

- Los tramos tienen una longitud igual a la longitud de referencia del tramo blanco.

- Los tramos son deslizantes, es decir, se van deslizando de 100 en 100 metros, de manera que un tramo i de longitud igual a la de referencia es seguido por un tramo i+1 que comienza 100 metros después del tramo i y acaba 100 metros después de dónde acaba el tramo i. Si bien esta circunstancia introduce una correlación espacial entre los tramos, análisis posteriores han demostrado que el modelo generado se ajusta bien a la explicación de los Tramos Blancos identificados.

El tamaño de la muestra inicial (con todas las categorías de vías) ascendía a 30.792 registros que, al considerar exclusivamente las categorías $\mathrm{B}$ y $\mathrm{C}$, quedan reducidos a 22.754 registros.

A cada tramo, según el criterio definido anteriormente, se le añaden los datos relativos a las siguientes variables (a cada tramo se le atribuye, para cada variable, el promedio de los valores disponibles; se analizó, así mismo, la posibilidad de incluir, para las variables de diseño geométrico, los valores que sólo superan el $15 \%$ o el $85 \%$ de los registros, si bien los resultados obtenidos fueron muy similares a los generados con los valores promedio):

- Existencia de tramo blanco; se refleja en la base de datos con el valor «SI» $(=1)$; consecuentemente, figura «NO» $(=0)$ cuando no existe tramo blanco. Es la variable dependiente.

- Tráfico: Intensidad Media Diaria.

- Tráfico de vehículos pesados: tanto la Intensidad Media Diaria de vehículos pesados como el porcentaje sobre el tráfico total.

- Velocidad media de circulación.-

- Accidentes con heridos graves o víctimas mortales.

- Número de fallecidos.

- Número de heridos graves.

- Exposición: tráfico en millones de vehículos - kilómetro.

- Índice de peligrosidad: relación entre el número de accidentes que se han producido en los 5 años (2006-2010) y el tráfico en esos 5 años en millones de vehículos - kilómetro.

- Índice de mortalidad: relación entre el número de víctimas mortales que se han producido en los 5 años (2006-2010) y el tráfico en esos 5 años en millones de vehículos - kilómetro.
Asimismo, se añaden las siguientes variables relativas a la geometría de la vía, obtenidas del inventario de carreteras realizado por el Ministerio de Fomento entre 2006 y 2009; para cada uno de estos valores se calcula el promedio en el tramo:

- Número de carriles

- Anchura del arcén derecho

- Anchura del arcén izquierdo

- Valor absoluto del radio de curvatura

- Valor absoluto del peralte

- Valor absoluto de la pendiente

- Ancho de calzada principal

- Ancho de plataforma

- Ancho de carril (obtenido a partir de los datos de ancho de calzada y número de carriles)

De cara a evitar la dependencia y duplicidad de las variables consideradas, no se introducen en el análisis las siguientes variables:

- Número de accidentes con víctimas, fallecidos y heridos graves, por estar implícitamente consideras en la existencia o no de tramo blanco.

- Exposición, por estar incluida en la variable de intensidad media de tráfico y la consideración de las longitudes de los tramos.

- Índices de peligrosidad y mortalidad, por estar relacionadas con los parámetros expuestos anteriormente (accidentes, víctimas mortales y niveles de tráfico).

El análisis supone manejar más de 340.000 datos.

La caracterización de las variables, una vez eliminados los extremos de las mismas, se incluyen en la Tabla 2. En la Tabla 3 se incluyen los resultados del cálculo de correlaciones entre variables, que demuestra que no existe una fuerte correlación entre la variable dependiente (existencia de tramo blanco) y el resto de las variables; se aprecia, asimismo, correlación entre las variables de tráfico total y tráfico de vehículos pesados, por lo que no entran simultáneamente en el modelo.

De cara a conocer si existen diferencias estadísticamente significativas en la variable dependiente motivadas por cada una de las variables independientes, se utiliza la prueba de contraste T de Student, que comprueba la hipótesis nula de que no existen diferencias estadísticamente significativas en la variable dependiente provocadas por la variable independiente; si el parámetro es estadísticamente significativo $(\mathrm{p}<0,05)$ se rechaza la hipótesis nula, asumiendo que existen diferencias. Para este caso, se observa que, para los dos grupos de registros considerados, todas las variables independientes generan diferencias estadísticamente significativas en la variable dependiente. Esta prueba permite rechazar la hipótesis nula de que las medias de ambos grupos (Tramos Blancos y tramos no blancos) son iguales, constatando la existencia de diferencias significativas entre los valores de las variables independientes en ambos casos.

\subsection{Definición del modelo estadístico para la identificación de tramos blancos.}

El análisis estadístico de la accidentalidad en carretera suele realizar con modelos multivariantes, tras los primeros modelos de regresión lineal univariantes, que arrojaron resultados 
Tabla 2. Estadísticos descriptivos de las variables del modelo. (Fuente: elaboración propia a partir de los datos de accidentalidad e inventario de carreteras facilitados para la investigación).

\begin{tabular}{|l|c|c|c|c|c|c|c|c|c|c|c|c|c|}
\hline \multicolumn{10}{|c|}{} \\
\hline
\end{tabular}

Tabla 3. Correlaciones entre las variables. (Fuente: elaboración propia a partir de los datos de accidentalidad e inventario de carreteras facilitados para la investigación).

\begin{tabular}{|c|c|c|c|c|c|c|c|c|c|c|c|c|c|c|c|}
\hline \multicolumn{16}{|c|}{ Correlaciones } \\
\hline & & $\begin{array}{c}\text { Existencia } \\
\text { de tramo } \\
\text { blanco }\end{array}$ & $\begin{array}{c}\text { Tráfico } \\
\text { total } \\
\text { (vehículos } \\
\text { diarios) }\end{array}$ & $\begin{array}{c}\text { Tráfico } \\
\text { pesados } \\
\text { (vehículos } \\
\text { diarios) }\end{array}$ & $\begin{array}{c}\% \\
\text { vehículos } \\
\text { pesados }\end{array}$ & $\begin{array}{c}\text { Velocidad } \\
(\mathrm{km} / \mathrm{h})\end{array}$ & $\begin{array}{c}\text { No } \\
\text { carriles }\end{array}$ & $\begin{array}{l}\text { Ancho } \\
\text { arcén } \\
\text { drch. } \\
(\mathrm{m})\end{array}$ & $\begin{array}{c}\text { Ancho } \\
\text { arcén } \\
\text { izqd. } \\
\text { (m) }\end{array}$ & $\begin{array}{l}\text { Ancho } \\
\text { carril } \\
\text { principal } \\
\text { (m) }\end{array}$ & $\begin{array}{c}\text { Ancho } \\
\text { calzada } \\
\text { principal } \\
\text { (m) }\end{array}$ & $\begin{array}{c}\text { Ancho } \\
\text { plataforma } \\
\text { principal } \\
\text { (m) }\end{array}$ & $\begin{array}{c}\text { Radio } \\
\text { (m) }\end{array}$ & $\begin{array}{l}\text { Peralte } \\
\text { (\%) }\end{array}$ & $\begin{array}{c}\text { Pendiente } \\
(\%)\end{array}$ \\
\hline \multirow{2}{*}{$\begin{array}{c}\text { Existencia } \\
\text { de tramo } \\
\text { blanco }\end{array}$} & $\begin{array}{l}\begin{array}{l}\text { Correlación } \\
\text { de Pearson }\end{array} \\
\end{array}$ & 1 &,$- 284^{* *}$ &,$- 236^{* *}$ &,$- 086^{* *}$ &,$- 245^{* *}$ &, $051^{* *}$ &, $081^{* *}$ &,$- 117^{* *}$ & ,001 & , $064^{*+*}$ &, $046^{* *}$ &, $089^{* *}$ &, $032^{* *}$ &, $015^{*}$ \\
\hline & Sig. (bilateral) & & 0,000 & , ooo & , ooo & , ooo & , ooo & , 000 & , ooo & ,860 & , 000 & , 000 & , ooo & , ooo & ,033 \\
\hline \multirow{2}{*}{$\begin{array}{c}\text { Tráfico } \\
\text { total }\end{array}$} & $\begin{array}{l}\text { Correlación } \\
\text { de Pearson }\end{array}$ &,$- 284^{* *}$ & 1 &, $853^{* *}$ &, $120^{* *}$ &, $233^{* *}$ &, $050^{* *}$ &,$- 165^{* *}$ &, $175^{* *}$ &, $095^{* *}$ &, $062^{* *}$ & , $045^{* *}$ &,$- 220^{* *}$ &,$- 026^{* *}$ &, $204^{* *}$ \\
\hline & Sig. (bilateral) & 0,000 & & 0,000 & , & , ooo & , ooo & , 000 & ,ooo & , ooo & , 000 & , ooo & , ooo & , ooo & , ooo \\
\hline \multirow{2}{*}{$\begin{array}{l}\text { Tráfico } \\
\text { pesados }\end{array}$} & $\begin{array}{l}\text { Correlación } \\
\text { de Pearson }\end{array}$ &,$- 236^{* *}$ &, $853^{* *}$ & 1 &, $541^{* *}$ &, $124^{* *}$ &, $077^{* *}$ &,$- 272^{* *}$ &,$- 050^{* *}$ &, $086^{* *}$ &, $078^{* *}$ &,$- 070^{* *}$ &,$- 296^{* *}$ &, $021^{* *}$ &, $277^{* *}$ \\
\hline & Sig. (bilateral) & , ,000 & 0,000 & & 0,000 & , ooo & , ooo & 0,000 & ,ooo & , ooo & , 000 & , ooo & 0,000 & ,003 & 0,000 \\
\hline \multirow{2}{*}{$\begin{array}{c}\% \\
\text { Pesados }\end{array}$} & $\begin{array}{l}\text { Correlación } \\
\text { de Pearson }\end{array}$ &,$- 086^{* *}$ & , $120^{* *}$ &, $541^{* *}$ & 1 &,$- 143^{* * *}$ &, $056^{*+}$ &,$- 196^{* *}$ &,$- 323^{* *}$ &, $077^{* *}$ &, $086^{* *}$ &,$- 131^{* *}$ &,$- 241^{* * *}$ & ,006 &, $211^{*+}$ \\
\hline & Sig. (bilateral) & , ооo & , о०о & 0,000 & & , оoo & , оoo & , 000 & 0,000 & , ooo & , ooo & , ooo & , ooo & ,403 & , ooo \\
\hline \multirow{2}{*}{ Velocidad } & $\begin{array}{l}\text { Correlación } \\
\text { de Pearson }\end{array}$ &,$- 245^{* *}$ &, $233^{* * *}$ &, $124^{* *}$ &,$- 143^{* *}$ & 1 &, $037^{* *}$ &,$- 628^{* *}$ &, $208^{* *}$ &,$- 316^{* *}$ &,$- 136^{* *}$ &,$- 311^{* *}$ &,$- 280^{* * *}$ &, $089^{* * *}$ &, $021^{* *}$ \\
\hline & Sig. (bilateral) & ,ooo & , ooo & , ooo & , ooo & & , ooo & 0,000 & ,ooo & 0,000 & , ooo & 0,000 & 0,000 & ,ooo & ,004 \\
\hline \multirow{2}{*}{$\begin{array}{c}\mathrm{N}^{\mathrm{a}} \\
\text { carriles }\end{array}$} & $\begin{array}{l}\text { Correlación } \\
\text { de Pearson }\end{array}$ & ,051 &, $050^{* *}$ & , $077^{* *}$ &, $056^{* *}$ &, $037^{* *}$ & 1 &,$- 159^{* *}$ &,$- 100^{* *}$ &,$- 077^{* *}$ &, $820^{* *}$ &, $592^{* *}$ &,$- 229^{* *}$ &, $196^{* *}$ &, $302^{* *}$ \\
\hline & Sig. (bilateral) & , ooo & , о0о & , o0o & , ooo & , ooo & & , ooo & , ooo & , ooo & 0,000 & 0,000 & , ooo & , oоo & 0,000 \\
\hline \multirow{2}{*}{$\begin{array}{c}\text { Arcén } \\
\text { derecho }\end{array}$} & $\begin{array}{l}\text { Correlación } \\
\text { de Pearson }\end{array}$ &, $081^{* *}$ &,$- 165^{* *}$ &,$- 272^{* *}$ &,$- 196^{* *}$ &,$- 628^{* *}$ &,$- 159^{* *}$ & 1 &, $264^{* *}$ &, $288^{* *}$ & ,007 &, $537^{+*}$ &, $433^{* *}$ &,$- 174^{* *}$ &,$- 234^{* *}$ \\
\hline & Sig. (bilateral) & , 000 & , ooo & 0,000 & , ooo & 0,000 & , ooo & & , ooo & 0,000 &, 324 & 0,000 & 0,000 & , ooo & , ooo \\
\hline \multirow{2}{*}{$\begin{array}{c}\text { Arcén } \\
\text { izquierdo }\end{array}$} & $\begin{array}{l}\text { Correlación } \\
\text { de Pearson }\end{array}$ &,$- 117^{* *}$ &, $175^{* *}$ &,$- 050^{* * *}$ &,$- 323^{* *}$ & ,208** &,$- 100^{* *}$ &, $264^{* *}$ & 1 &,$- 225^{* *}$ &,$- 217^{* *}$ & ,299** &, $188^{* *}$ &,$- 245^{* *}$ &,$- 217^{* *}$ \\
\hline & Sig. (bilateral) & , ooo & , о0о & , оoo & 0,000 & , ooo & , 000 & , 000 & & , ooo & , o00 & 0,000 & , ooo & , ooo & , 000 \\
\hline \multirow{2}{*}{$\begin{array}{c}\text { Ancho } \\
\text { carril } \\
\text { principal }\end{array}$} & \begin{tabular}{|c|}
$\begin{array}{c}\text { Correlación de } \\
\text { Pearson }\end{array}$ \\
\end{tabular} & ,001 &, $095^{* *}$ &, $086^{* *}$ & , $077^{* *}$ &,$- 316^{* *}$ &,$- 077^{* *}$ &, $288^{* *}$ &,$- 225^{*+}$ & 1 &, $360^{* * *}$ &, $348^{* *}$ &, $059^{* *}$ &,- 002 &, $018^{*}$ \\
\hline & Sig. (bilateral) & ,860 & , о०о & , оoo & , о00 & 0,000 & , ooo & 0,000 & ,ooo & & 0,000 & 0,000 & ,ooo &, 832 & ,014 \\
\hline \multirow{2}{*}{$\begin{array}{c}\text { Ancho } \\
\text { calzada } \\
\text { principal }\end{array}$} & \begin{tabular}{|c|}
$\begin{array}{c}\text { Correlación de } \\
\text { Pearson }\end{array}$ \\
\end{tabular} &, $064^{* *}$ &, $062^{* * *}$ &, $078^{* * *}$ & ,o86 &,$- 136^{* *}$ &, $820^{* *}$ &, 007 &,$- 217^{* *}$ &, $360^{* *}$ & 1 &, $773^{* *}$ &,$- 161^{* *}$ &, $225^{* *}$ & ,291** \\
\hline & Sig. (bilateral) & ,ooo & , 000 & ,ooo & , ooo & , ooo & o,ooo &, 324 & ,ooo & 0,000 & & 0,000 & , ooo & , ooo & 0,000 \\
\hline \multirow{2}{*}{$\begin{array}{c}\text { Ancho } \\
\text { plataforma } \\
\text { principal }\end{array}$} & \begin{tabular}{|c|}
$\begin{array}{c}\text { Correlación de } \\
\text { Pearson }\end{array}$ \\
\end{tabular} &, $046^{* *}$ & , $045^{*+}$ &,$- 070^{* *}$ &,$- 131^{* *}$ &,$- 311^{* *}$ &, $592^{* *}$ &, $537^{*+}$ & ,299 &, $348^{* *}$ &, $773^{* *+}$ & 1 &, $120^{* * *}$ &, $025^{* *}$ &, $066^{* *}$ \\
\hline & Sig. (bilateral) & , ooo & , 000 & , ooo & , ooo & 0,000 & o,ooo & 0,000 & 0,000 & 0,000 & 0,000 & & ,ooo & , ooo & ,ooo \\
\hline \multirow{2}{*}{ Radio } & \begin{tabular}{|c|}
$\begin{array}{c}\text { Correlación de } \\
\text { Pearson }\end{array}$ \\
\end{tabular} &, $089^{* *}$ &,$- 220^{* *}$ &,$- 296^{+*}$ &,$- 241^{+*}$ &,$- 280^{* * *}$ &,$- 229^{* * *}$ &, $433^{+*}$ &, $188^{* *}$ &, $059^{* *}$ &,$- 161^{* *}$ & ,120 & 1 &,$- 636^{* *}$ &,$- 695^{* *}$ \\
\hline & Sig. (bilateral) & , ooo & , 000 & 0,000 & , ooo & 0,000 & , ooo & 0,000 & , ooo & , оoo & , ooo & , ooo & & 0,000 & 0,000 \\
\hline \multirow{2}{*}{ Peralte } & \begin{tabular}{|c|}
$\begin{array}{c}\text { Correlación de } \\
\text { Pearson }\end{array}$ \\
\end{tabular} &, $032^{* *}$ &,$- 026^{* *}$ & ,021** & , o06 &, $089^{* *}$ &, $196^{* *}$ &,$- 174^{* *}$ &,$- 245^{* *}$ &,- 002 &, $225^{* *}$ &, $025^{* * *}$ &,$- 636^{* *}$ & 1 &, $587^{* *}$ \\
\hline & Sig. (bilateral) & , ooo & , 000 & ,003 & ,403 & , ooo & ,ooo & , 000 & , ooo &, 832 & , ooo & , ooo & 0,000 & & 0,000 \\
\hline \multirow{2}{*}{ Pendiente } & \begin{tabular}{|c|}
$\begin{array}{c}\text { Correlación de } \\
\text { Pearson }\end{array}$ \\
\end{tabular} &, $015^{*}$ & ,204 & $277^{* *}$ &, $211^{* *}$ &, $021^{* *}$ &, $302^{* *}$ &,$- 234^{* *}$ &,$- 217^{* *}$ &, $018^{*}$ &, $291^{* *}$ &, $066^{* *}$ &,$- 695^{* *}$ &, $587^{* *}$ & 1 \\
\hline & Sig. (bilateral) & , 033 & , ooo & 0,000 & , ooo & ,004 & o,ooo & , ooo & ,ooo & ,014 & 0,000 & ,ooo & 0,000 & 0,000 & \\
\hline
\end{tabular}

** La correlación es significativa al nivel o,01 (bilateral)

* La correlación es significante al nivel o,05 (bilateral)

poco satisfactorios en muchos casos. Existe un consenso en el ámbito de la investigación en seguridad vial acerca de la modelización de los accidentes, que asume que los modelos lineales generales con las distribuciones de Poisson o binomial negativa son apropiados para modelar la frecuencia o tasa de accidentes (26). Miaou (27) realizó una comparación entre estos modelos, concluyendo la idoneidad de ambos para modelar accidentes, y recomendando la distribución binomial negativa para casos de sobredispersión de los datos. Desde entonces, numerosas investigaciones han aplicado estos procedimientos, que han sido valorados junto con otros modelos (28). 
Mediante el uso de estos modelos, se han realizado multitud de investigaciones para predecir los niveles de accidentalidad asociados a características de la geometría de la vía y variables de tráfico. Existen referencias a las dificultades metodológicas de la aplicación de los modelos de regresión múltiple, debidas a la variabilidad propia del fenómeno de la accidentalidad y a la gran cantidad de factores que influyen en él, que se consideran asociados a los índices de determinación bajos que suelen presentar estos modelos (29).

Cuando la variable dependiente es de tipo cualitativo, se aplican con frecuencia los modelos logísticos para evaluar la contribución de factores de influencia sobre variables como la severidad de los accidentes de tráfico; Dominguez (30) desarrollo un modelo de regresión logística binario para la estimación de la gravedad de los accidentes por salida de calzada. En la regresión de tipo binario, la variable dependiente puede tomar el valor de 1 con una probabilidad de suceso p, o el valor de o con la probabilidad 1-p. En una regresión logística, la relación entre la variable dependiente y las variables independientes no es una función de tipo lineal; la estructura de un modelo de regresión logística binaria figura en las ecuaciones [1] y [2] es (31):

$$
p(Y=1)=\frac{1}{1+\mathrm{e}^{-Z}}
$$

Siendo $Z$ :

$$
Z=\propto+\beta_{1} x_{1}+\beta_{2} x_{2}+\ldots+\beta_{k} x_{k}
$$

La formulación de un modelo de regresión logística binario para las autopistas de peaje consideradas se realizó considerando como variable dependiente la existencia de tramo blanco (1: existe tramo blanco; o: no existe tramo blanco), mientras que como variable independiente se consideraron la Intensidad Media Diaria de Tráfico total (IMD), la Intensidad Media Diaria de Tráfico de vehículos pesados (IMD $)$ el porcentaje de vehículos pesados (PP), la velocidad media de circulación (VM), el número de carriles (NC), la anchura del arcén derecho (AAD), la anchura del arcén izquierdo (AAI), el ancho de carril (AC), el valor absoluto del radio de curvatura (RC),el valor absoluto del peralte (PR), el valor absoluto de la pendiente (P), el ancho de calzada principal (ACP) y el ancho de la plataforma principal (APP).

En una primera aproximación, se realizó un modelo de regresión logística binario para la totalidad de los tramos definidos de las autopistas de peaje a las que se ha hecho referencia, independientemente del nivel de tráfico; el modelo obtuvo unos resultados muy limitados en cuanto al porcentaje de casos de existencia o no de tramo blanco clasificados correctamente. Ante esta situación, se decidió realizar una división de tramos en función de la intensidad media diaria, por medio de un análisis cluster, que permitió establecer diferencias de grupos según la IMD. Así, se dividió la muestra en tres grandes grupos:

- Grupo 1: IMD inferior a 10.000 vehículos al día (6264 registros)

- Grupo 2: IMD entre 10.000 y 20.000 vehículos al día (8085 registros)

- Grupo 3: IMD superior a 20.000 vehículos al día (5053 registros)

A continuación se realizó un análisis de varios modelos logísticos binarios para cada uno de los grupos definidos anteriormente; mientras que para el grupo 1 y grupo 3 los modelos arrojaron unos resultados pobres en cuanto a clasificación correcta de los casos, en el grupo 2 se obtuvieron resultados más favorables.

De entre todos los modelos desarrollados para los niveles de tráfico entre 10.000 y 20.000 vehículos diarios, que incluían todas las variables en el estudio, el que arrojó mejores resultados, en términos de clasificación correcta de los casos y análisis de sensibilidad es el que se incluye a continuación en la Tabla 4.

El resto de las variables queda fuera del modelo en este análisis provisional, por no ser significativas.

Así, el parámetro $Z$ se define de la siguiente manera en la ecuación [3]:

$$
\begin{gathered}
Z=154.335+6.839 * A C P-0.022 * I M D p+ \\
+106.33 * P P-0.967 * V M-23.072 * N C- \\
-6.421 * A A D-6.866 * A C
\end{gathered}
$$

Donde ACP es el Ancho de la Calzada Principal, $\mathrm{IMD}_{\mathrm{p}}$ es la Intensidad Media Diaria de vehículos pesados, PP es el Porcentaje de vehículos Pesados, VM es la Velocidad Media de circulación, NC es el Número de Carriles, AAD es la Anchura del Arcén Derecho y AC es el Ancho de Carril.

Y la probabilidad de encontrar un tramo blanco puede formu-

\begin{tabular}{|c|c|c|c|c|c|}
\hline & B & E.T. & Wald & gl & Sig. \\
\hline Velocidad media de circulación (VM) &,- 967 & ,044 & 473,130 & 1 & , ooo \\
\hline Número de carriles (NC) & $-23,072$ & 2,688 & 73,695 & 1 & ,000 \\
\hline Ancho del arcén derecho (AAD) & $-6,421$ & ,434 & 219,334 & 1 & ,000 \\
\hline Ancho de la calzada principal (ACP) & 6,839 & ,676 & 102,330 & 1 & ,000 \\
\hline Ancho de carril (AC) & $-6,866$ & 1,787 & 14,765 & 1 & , ooo \\
\hline \% de vehículos pesados (PP) & 106,330 & 5,382 & 390,353 & 1 & ,000 \\
\hline $\begin{array}{l}\text { Tráfico de vehículos pesados total } \\
\text { (intensidad media diaria) }\left(\operatorname{IMD}_{\mathrm{p}} \text { ) }\right.\end{array}$ &,- 022 & ,001 & 495,947 & 1 & ,000 \\
\hline Constante & 154,335 & 8,893 & 301,161 & 1 & ,000 \\
\hline
\end{tabular}
larse como aparece en la ecuación [4]:

$$
P=\frac{1}{1+e^{-154.335-6.639^{*} A C P+0.022^{*} I M D p-106.33^{*} P P+0.967^{*} V M+23.072^{*} N C+6.42^{*} A A D+6.866^{*} A C}}
$$

Tabla 4. Conclusiones del modelo de regresión logística binario. (Fuente: elaboración propia). 
En la Tabla 5 se resumen las características del modelo.

Tabla 5. Resumen del modelo de regresión logística binario. (Fuente: elaboración propia).

\begin{tabular}{|c|c|c|}
\hline \multicolumn{3}{|c|}{ Resumen del modelo } \\
\hline $\begin{array}{c}-2 \text { log de la } \\
\text { verosimilitud }\end{array}$ & $\begin{array}{c}\text { R cuadrado de Cox } \\
\text { y Snell }\end{array}$ & $\begin{array}{c}\text { R cuadrado de } \\
\text { Nagelkerke }\end{array}$ \\
\hline 3130,597 &, 303 &, 569 \\
\hline
\end{tabular}

Si bien el estadístico «-2 log de la verosimilitud» obtenido en el modelo es elevado, presenta una notable reducción respecto a los modelos que introducen otras variables. El estadístico «R cuadrado de Cox y Snell» y el estadístico «R cuadrado de Nagelkerke», versión corregida del anterior, hace referencia a la capacidad del modelo para explicar la variación de la variable dependiente; el valor obtenido hace referencia a que las variables independientes explican aproximadamente el $30 \%$ de la existencia de tramo blanco.

Sin embargo, los resultados concluyen provisionalmente que el modelo predice de manera correcta el 91,1\% de las observaciones, repartidas en un 96,4 \% de predicción correcta en los tramos no blancos y un 54,5 \% de predicción correcta en los tramos blancos.

\section{APLICACIÓN A LAS AUTOPISTAS DE PEAJE ESPAÑOLAS}

La aplicación del modelo definido a datos reales permite realizar el análisis de sensibilidad de las siguientes figuras (Figura 1), (Figura 2), (Figura 3).

Se observa que, para este tipo de autopistas, la presencia de tramos blancos se produciría, según los resultados obtenidos en un primer análisis, con mayor frecuencia bajo las siguientes circunstancias:

- Por encima de $110 \mathrm{~km} / \mathrm{h}$ de velocidad media de circulación, se reduciría notablemente la probabilidad de encontrar un tramo blanco.

- En vías con anchos de calzada superiores a 9,5 metros, se encontrarían tramos blancos con mayor frecuencia, mientras que por debajo de 8 metros parecen ser menos frecuentes.

- La intensidad del tráfico de vehículos pasados parece tener una relación con la presencia de tramos blancos; de esta manera, con más de 1.000 vehículos pesados al día, no aparecen tramos blancos según los análisis realizados.

\section{CONCLUSIONES Y LÍNEAS DE INVESTIGACIÓN FUTURAS}

Incluso en una red de carreteras de las más altas prestaciones, como es la red de autopistas de peaje, que se caracteriza por una coherencia y consistencia en el diseño, entendida como el grado de adecuación entre el comportamiento de la carretera y lo que el conductor espera de la misma (32), parece posible encontrar diferencias en parámetros específicos del diseño de las vías que dan lugar a la aparición de tramos sin accidentes que no parecen ser perturbaciones estadísticas.

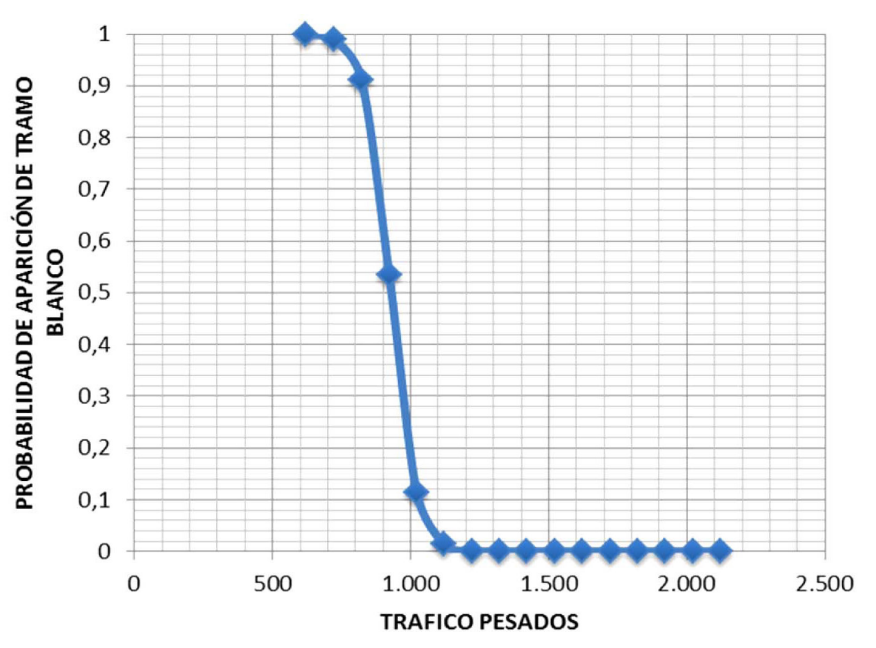

Figura 1. Probabilidad de encontrar un tramo blanco según el modelo, al modificar la variable TRÁfICO DE VEHículos PESADOS (IMDp) y mantener constantes el resto de las variables.

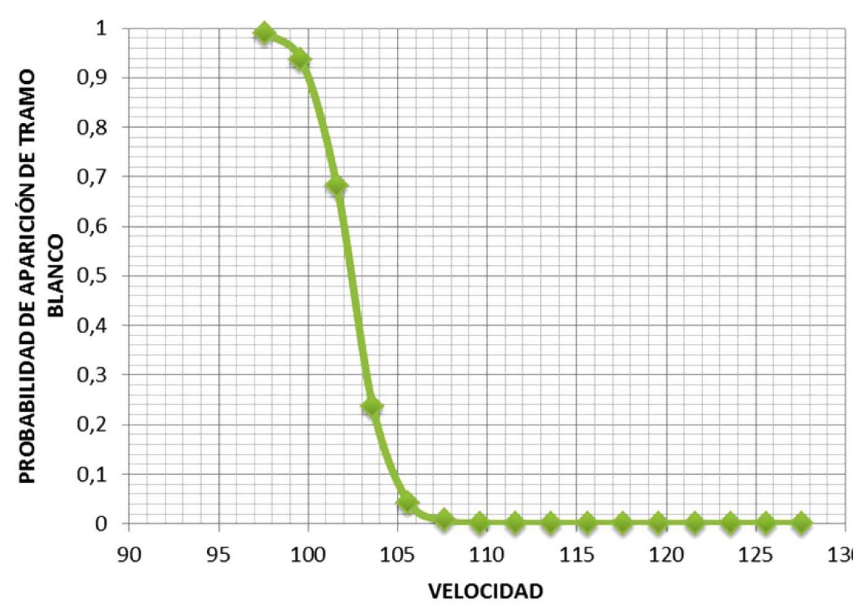

Figura 2. Probabilidad de encontrar un tramo blanco según el modelo, al modificar la variable Velocidad MEdia DE Circulación (VM) y mantener constantes el resto de las variables.

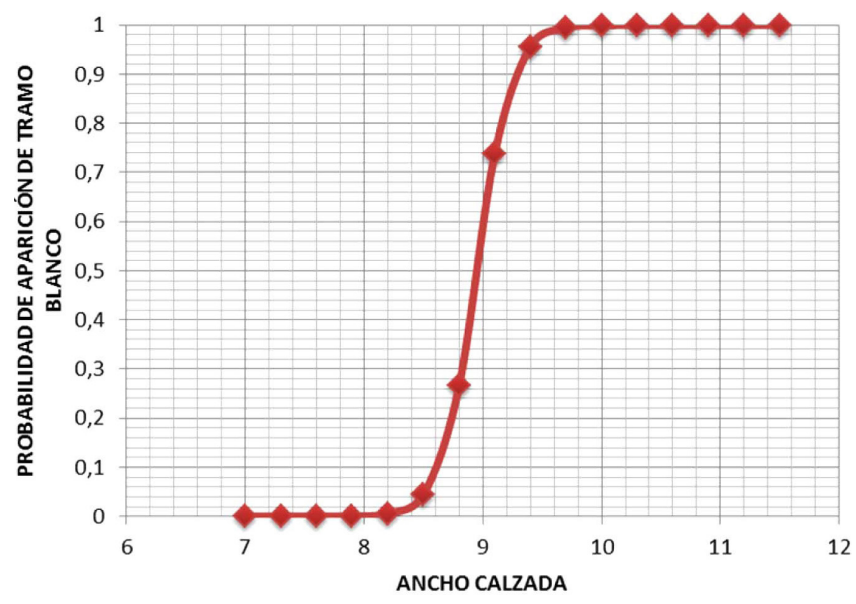

Figura 3. Probabilidad de encontrar un tramo blanco según el modelo, al modificar la variable ANCHO DE CALZADA PRINCIPAL (ACP) y mantener constantes el resto de las variables.

Los resultados provisionales del modelo demuestran que, en situaciones similares de parámetros generales de geometría de la vía y de tráfico, tanto de vehículos totales como de vehículos pesados, bajo determinadas circunstancias relativas a 
parámetros específicos, existiría una mayor tendencia a que se presenten tramos sin accidentes de suficiente longitud, durante un periodo de tiempo de cinco años.

De cara a la construcción de nuevas autopistas y la explotación de las existentes, se sugiere evitar situaciones que permitan una conducción a elevadas velocidades, y asegurar la existencia de espacio suficiente en la calzada, con una anchura de arcén amplia; la presencia de vehículos pesados parece ser un parámetro influyente en el hecho de que se presenten Tramos Blancos, por lo que se sugiere investigar en mayor profundidad los efectos del aumento de este tipo de tráfico en autopistas de peaje, especialmente en la situación actual, en la que se está promoviendo un trasvase de tráfico en este sentido por motivos de fluidez y seguridad.

Si bien los estadísticos que definen el ajuste del modelo están lejos de ser óptimos, los resultados provisionales apuntan a una línea de investigación de interés para generar nuevas visiones acerca de la seguridad de las infraestructuras viarias. En carreteras con altos estándares de diseño, como son las autopistas de peaje, donde no se presentan, en términos generales, puntos con diseños defectuosos, la aleatoriedad de los accidentes es mayor que en vías convencionales, por lo que el hecho de encontrar los denominados «tramos blancos» se asume a priori como un hecho poco frecuente.
Los resultados obtenidos deben considerarse como la conclusión de un análisis provisional, dado que, indudablemente, existen otros parámetros, tanto de diseño de la vía como de la circulación, el entorno, el factor humano o el vehículo que podrían tener una influencia en el hecho que se analiza, y no se han considerado por no disponer de esta información. Las limitaciones en el manejo de los datos, incluyendo la falta de información más detallada sobre intensidad de tráfico, velocidades puntuales, datos de climatología, estado de conservación de los firmes, características de los márgenes de las vías (dada la elevada representatividad de los accidentes por salida de vía en el total de accidentes que se producen en las carreteras), etc., disminuyen las posibilidades de obtener resultados más fiables y detallados. Sin embargo, se ha conseguido extraer algunas conclusiones que pueden ser de interés en el ámbito de la investigación en seguridad vial, aun teniendo en cuenta las limitaciones expuestas. Los posibles problemas de correlaciones espaciales han sido contemplados en estudios posteriores sin que se hayan encontrado razones para modificar las conclusiones alcanzadas.

Las posibilidades de mejora del modelo son múltiples y van desde el análisis de otro tipo de vías, la profundización en la implicación de los parámetros de tráfico en la presencia de tramos blancos o la consideración de condicionantes climatológicos, entre otros.

\section{REFERENCIAS}

(1) OMS. (2013). Informe sobre la situación mundial de la seguridad vial 2013. Apoyo al Decenio de Acción. Ginebra: Organización Mundial de la Salud.

(2) Comisión Europea. (2001). Libro blanco. La política Europea de transportes de cara al 2010: la hora de la verdad. Bruselas: Comisión Europea.

(3) Comisión Europea. (2010). Comunicación de la Comisión al Parlamento Europeo, al Consejo, al Comité Económico y Social Europeo y al Comité de las Regiones. Hacia un espacio europeo de seguridad vial: orientaciones políticas sobre seguridad vial 2011-2020. Bruselas: Comisión Europea.

(4) Zaragoza, A. (2005). La teoría de la homeóstasis del riesgo subjetivo. Carreteras, 142: 21-34.

(5) Parlamento Europeo y Consejo de la Unión Europea. (2008). Directiva Europea 2008/96/CE de 19 de Noviembre de 2008 sobre gestión de la seguridad de las infraestructuras viarias. Diario Oficial de la Unión Europea, $\mathrm{n}^{0} 319$.

(6) Ministerio de Fomento. (2011). Real Decreto 345/2011, de 11 de marzo, sobre gestión de la seguridad de las infraestructuras viarias en la Red de Carreteras del Estado. Boletín Oficial del Estado, no 61. España.

(7) Mohamedhah, Y. M., Paniati, J. F., Hobeika, A.G. (1993). Truck accident models for interstate and two lane rural roads. Transportation Research Record, 1407: 35-41.

(8) Garber, N.J., Joshua, S.C. (2000.) Effect of speed, flow and geometric characteristics on crash frequency for two lane highways. Transportation Research Record, 1717: 76-83, doi: http://dx.doi.org/10.3141/1717-10.

(9) Bauer, K.M., Harwood, D. (2000). Statistical models of At-Grade Intersection accidents Addendum. Publication FHWA$R D$-99-094. Federal Highway Administration.

(10) Karlaftis, M.G., Golias, I. (2002). Effects of road geometry and traffic volumes on rural roadway accidents. Accident Analysis and Prevention, 34(3): 357-365, doi: http://dx.doi.org/10.1016/Sooo1-4575(01)ooo33-1.

(11) Hiselius, L.W. (2004). Estimating the relationship between accident frequency and homogeneous and inhomogeneous traffic flows. Accident Analysis and Prevention, 36(6): 985-992, doi: http://dx.doi.org/10.1016/j.aap.2003.11.002.

(12) Arenas, B. (2008). Desarrollo de una metodología para la evaluación de la seguridad de tráfico y el análisis de la influencia del transporte de mercancías y el trasvase intermodal entre modos terrestres (Tesis doctoral). Madrid: Universidad Politécnica.

(13) Deublein, M., Schubert, M., Adey, B., Köhler, J., Faber, M. (2013). Prediction of road accidents: A Bayesian Hierarchical approach. Accident Analysis and Prevention, 51: 274-291, doi: http://dx.doi.org/10.1016/j.aap.2012.11.019.

(14) Hadi, M.A., Aruldhas, J., Chow, L.F., Wattleworth, J.A. (1995). Estimating safety effects of cross-section design for various highway types using negative binomial regression. Transportation Research Board, 1500: 169-177.

(15) Karlaftis, M.G., Tarko, A.P. (1998). Heterogeneity considerations in accident modelling. Accident Analysis and Prevention, 30(4): 425-433, doi: http://dx.doi.org/10.1016/Soo01-4575(97)00122-X.

(16) Abdelaty, M., Radwan, A.E. (2000). Modelling traffic accident occurrence and involvement. Accident Analysis and Prevention, 32(5): 633-642, doi: http://dx.doi.org/10.1016/Sooo1-4575(99)0oo94-9. 
(17) Xie, Y., Lord, D., Zhang, Y. (2007). Predicting motor vehicle collisions using Bayesian neural network models. An empirical analysis. Accident Analysis and Prevention, 39(5): 922-933, doi: http://dx.doi.org/10.1016/j.aap.2006.12.014.

(18) Vogt, A., Bared, J. (1998). Accident models for two-lane rural segments and intersections. Transportation Research Record, 1635: 18-29, doi: http://dx.doi.org/10.3141/1635-03.

(19) Persaud, B., Retting, R.A., Lyon, C. (2000). Guidelines for the identification of hazardous highway curves. Transportation Research Record, 1717: 14-18, doi: http://dx.doi.org/10.3141/1717-03.

(20) Chang, L.Y., Chen, W.C. (2005). Data mining of tree-based models to analyze freeway accident frequency. Journal of Safety Research, 36(4): 365-375, doi: http://dx.doi.org/10.1016/j.jsr.2005.06.013.

(21) Berhanu, G. (2004). Models relating traffic safety with roads environment and traffic flows on arterial roads in Addis Ababa. Accident Analysis and Prevention, 36(5): 697-704, doi: http://dx.doi.org/10.1016/j.aap.2003.05.002.

(22) Zaragoza, A,. Diaz, J., De la Peña, E. (2002, 11 de junio). Una nueva metodología en materia de seguridad vial: la identificación de los tramos sin accidentes. En V Congreso de Ingeniería de Transportes. Santander: Universidad de Cantabria.

(23) Diaz, J., De la Peña, E. (2003). Últimas tendencias en seguridad vial. Carreteras, 128: 6-16

(24) White Roads. http://www.whiteroads.eu/.

(25) Easy Way. https://www.easyway-its.eu/.

(26) Rangel, T. (2011). Evaluation of the effectiveness of safety-based incentives in Public Private Partnerships. Evidence from the case of Spain (Tesis doctoral). Madrid: Universidad Politécnica.

(27) Miaou, S.P. (1994). The relationaship between truck accients and geometric design of road sections: Possion versus negative binominal regressions. Accident Analysis and PRevention, 26(4): 471-482, doi: http://dx.doi.org/10.1016/ooo14575(94)90038-8.

(28) Lord, D., Mannering, F. (2010). The statistical analysis of crash-frequency data: a review and assessment of methodological alternatives. Transportation Research Part A, 44(5): 291-305, doi: http://dx.doi.org/10.1016/j.tra.2010.02.001.

(29) Pardillo, J. (2004) Procedimientos y de estudio, diseño y gestión de medidas de seguridad vial en las infraestructuras, p. 87. Madrid: Fundación Agustín de Betancourt.

(30) Dominguez, C.A. (2011). Caracterización de las condiciones de seguridad de las márgenes de carretera (Tesis doctoral). Madrid: Universidad Politécnica.

(31) Visauta, B. (1998). Análisis estadístico con SPSS para Windows, volumen II estadística multivariante, p. 52-69. Madrid: Mcgraw-Hill.

(32) García, A., Camacho, F. J. (2009). Evaluación de la seguridad vial de carreteras convencionales mediante la determinación de la consistencia global de su diseño geométrico. Carreteras, 163: 21-32. 